ITP-SB-96-21

April, 1996

\title{
Exact two-spinon dynamical correlation function of the Heisenberg model
}

\author{
A.H. Bougourzi ${ }^{1}$, M. Couture ${ }^{2}$ and M. Kacir $^{3}$ \\ ${ }^{1}$ Institute of Theoretical Physics \\ SUNY at Stony Brook \\ Stony Brook, NY 11794 \\ ${ }^{2}$ Neutron $\& 3$ Condensed Matter Sciences \\ AECL Research, Chalk River Laboratories \\ Chalk River, Ontario, Canada KOJ 1 JO \\ ${ }^{3}$ Service de Physique Theorique \\ CE-Saclay \\ F-91191 Gif-sur-Yvette Cedex, France
}

\begin{abstract}
We derive the exact contribution of two spinons to the dynamical correlation function of the spin-1/2 Heisenberg model. For this, we use the isotropic limits of the exact form factors that have been recently computed through the quantum affine symmetry of the anisotropic Heisenberg model $X X Z$.
\end{abstract}




\section{Introduction}

Ever since Niemeijer derived an exact expression for the dynamical correlation function (DCF) at any temperature of the spin-1/2 XY model [1], there has been a considerable amount of work in trying to extend his result to the more physically interesting case of the isotropic spin-1/2 Heisenberg model (i.e., $X X X$ model). For more details on the definitions of the spin chain models see Refs. [2, 3]. However, so far only approximate but very accurate results have been computed. For a comprehensive historic review, the importance of the DCF, an account of the existing results, and a list of references on this subject we recommend Refs. [4, 5, 6, 0, 8, 9]. In particular, in Ref. [5] an ansatz for the DCF at zero temperature was proposed based on Niemeijer's result and other approximate numerical and analytical results. But it has never been established whether this ansatz includes contributions from just two spinons or more. The spinon picture has been rigorously studied in the case of the Heisenberg model in [10], and is of great theoretical and experimental interest. Let us just mention that the main stumbling block in trying to compute the eaxct DCF for the Heisenberg model is due to the absence of exact results for the form factors. Unfortunately, so far the powerful method of the Bethe ansatz has not been able to provide them. The form factors are well understood now just in two-dimensional quantum field theories with familiar relativistic dispersion relations [11] but not yet in lattice models. However, an approach based on the concept of exact resolution of dynamics through just infinite-dimensional symmetries, and which is widely used in the context of string theory and conformal field theory, has been recently upgraded to the "massive" $X X Z$ model in [12, 13]. It provides almost all exact physical quantities (static correlation functions and form factors). Its only shortcomings are that it is based on a relatively complicated symmetry, which is the quantum affine algebra $U_{q}(\widehat{s l(2)})$, and that the physical quantities it leads to are somewhat complicated to deal with. To be more precise, they typically have a multi-integral form. Our main point in this paper is that all these latter problems disappear in the particular case of the two-spinon form factors of the Heisenberg model because they have a simpler form. Therefore we use them to compute the more interesting quantity of the exact two-spinon DCF at zero temperature.

Our letter is organized as follows: first we briefly review the results related to the diag- 
onalization of the anisotropic $X X Z$ Heisenberg model following Ref. [13]. Then we define the two-spinon DCF in the case of $X X Z$ in terms of the form factors of this model. Finally, we take the isotropic limit. As mentioned earlier, the crucial point is that considerable simplifications take place in this case due to the isotropy of the Heisenberg model, and thus allow us to derive a simple exact formula for DCF of two-spinon. We hope that the exact results contained in this paper will shed some new light on the spinon picture from both theoretical and experimental perspectives where already a considerable amount of work has been achieved over the past few decades.

\section{Diagonalization of the anisotropic Heisenberg model}

The Hamiltonian of the anisotropic (XXZ) Heisenberg model is defined by

$$
H_{X X Z}=-\frac{1}{2} \sum_{n=-\infty}^{\infty}\left(\sigma_{n}^{x} \sigma_{n+1}^{x}+\sigma_{n}^{y} \sigma_{n+1}^{y}+\Delta \sigma_{n}^{z} \sigma_{n+1}^{z}\right),
$$

where $\Delta=\left(q+q^{-1}\right) / 2$ is the anisotropy parameter. Here $\sigma_{n}^{x, y, z}$ are the usual Pauli matrices acting at the $n^{\text {th }}$ position of the formal infinite tensor product

$$
W=\cdots V \otimes V \otimes V \cdots
$$

where $V$ is the two-dimensional representation of $U_{q}(\operatorname{sl}(2))$ quantum group. We consider the model in the anti-ferromagnetic regime $\Delta<-1$, i.e., $-1<q<0$. The main point of Refs. [12, 13] is that the action of $H_{X X Z}$ on $W$ is not well defined due to the appearance

of divergences. However, this model is symmetric under the quantum group $U_{q}(\widehat{s l(2)})$, and therefore the eigenspace is identified with the following level $0 U_{q}(\widehat{s l(2)})$ module:

$$
\mathcal{F}=\sum_{i, j} V\left(\Lambda_{i}\right) \otimes V\left(\Lambda_{j}\right)^{*}
$$

where $\Lambda_{i}$ and $V\left(\Lambda_{i}\right) ; i=0,1$ are level $1 U_{q}(\widehat{s l(2)})$-highest weights and $U_{q}(\widehat{s l(2)})$-highest weight modules, respectively. Roughly speaking, $V\left(\Lambda_{i}\right)$ is identified with the subspace of the formal semi-infinite space

$$
X=\cdots V \otimes V \otimes V
$$

consisting of all linear combinations of spin configurations with fixed boundary conditions such that the eigenvalues of $\sigma_{n}^{z}$ are $(-1)^{i+n}$ in the limit $n \rightarrow-\infty$. The particle picture of 
this Hamiltonian is given in terms of vertex operators which act as intertwiners of $U_{q}(\widehat{s l(2)})$ modules, and which create the set of eigenstates (spinons) $\left\{\mid \xi_{1}, \cdots \xi_{n}>_{\epsilon_{1}, \cdots \epsilon_{n} ; i}, n \geq 0\right\}$. Here $i$ fixes the boundary conditions, $\xi_{j}$ are the spectral parameters living on the unit circle, and $\epsilon_{j}= \pm 1$ are the spins of the spinons. The actions of $H_{X X Z}$ and the translation operator $T$, which shifts the spin chain by one site, on $\mathcal{F}$ are given by

$$
\begin{aligned}
T \mid \xi_{1}, \cdots, \xi_{n}>_{i} & =\prod_{i=1}^{n} \tau\left(\xi_{i}\right)^{-1}\left|\xi_{1}, \cdots, \xi_{n}>_{1-i}, \quad T\right| 0>_{i}=\mid 0>_{1-i}, \\
H_{X X Z} \mid \xi_{1}, \cdots, \xi_{n}>_{i} & =\sum_{i=1}^{n} e\left(\xi_{i}\right) \mid \xi_{1}, \cdots, \xi_{n}>_{i},
\end{aligned}
$$

where

$$
\begin{aligned}
\tau(\xi) & =\xi^{-1} \frac{\theta_{q^{4}}\left(q \xi^{2}\right)}{\theta_{q^{4}}\left(q \xi^{-2}\right)}=e^{-i p(\alpha)}, \quad p(\alpha)=\operatorname{am}\left(\frac{2 K}{\pi} \alpha\right)-\pi / 2, \\
e(\xi) & =\frac{1-q^{2}}{2 q} \xi \frac{d}{d \xi} \log \tau(\xi)=\frac{2 K}{\pi} \sinh \left(\frac{\pi K^{\prime}}{K}\right) \operatorname{dn}\left(\frac{2 K}{\pi} \alpha\right) .
\end{aligned}
$$

Here, $e(\xi)$ and $p(\alpha)$ are the energy and the momentum of the spinon respectively, $\operatorname{am}(x)$ and $d n(x)$ are the usual elliptic amplitude and delta functions, with the complete elliptic integrals $K$ and $K^{\prime}$, and

$$
\begin{aligned}
q & =-\exp \left(-\pi K^{\prime} / K\right) \\
\xi & =i e^{i \alpha} \\
\theta_{x}(y) & =(x ; x)_{\infty}(y ; x)_{\infty}\left(x y^{-1} ; x\right)_{\infty}, \\
(y ; x)_{\infty} & =\prod_{n=0}^{\infty}\left(1-y x^{n}\right) .
\end{aligned}
$$

This means, $\sigma^{x, y, z}(t, n)$ at time $t$ and position $n$ are related to $\sigma^{x, y, z}(0,0)$ at time 0 and position 0 through:

$$
\sigma^{x, y, z}(t, n)=\exp \left(i t H_{X X Z}\right) T^{-n} \sigma^{x, y, z}(0,0) T^{n} \exp \left(-i t H_{X X Z}\right) .
$$

The completeness relation reads [13]:

$$
\mathbb{I}=\sum_{i=0,1} \sum_{n \geq 0} \sum_{\epsilon_{1}, \cdots, \epsilon_{n}= \pm 1} \frac{1}{n !} \oint \frac{d \xi_{1}}{2 \pi i \xi_{1}} \cdots \frac{d \xi_{n}}{2 \pi i \xi_{n}}\left|\xi_{n}, \cdots, \xi_{1}>_{\epsilon_{n}, \cdots, \epsilon_{1} ; i} \quad i_{i, \epsilon_{1}, \cdots, \epsilon_{n}}<\xi_{1}, \cdots, \xi_{n}\right| .
$$

\section{Two-spinon dynamical correlation function of the Heisenberg model}

First, we will define the dynamical correlation function we are considering in the case of the anisotropic Heisenberg model, where the particle picture is well understood and the form 
factors are known exactly [13]. However, let us note that the expressions of these form factors are very complicated to lead to a closed formula for the DCF in the anisotropic case. But, in the isotropic limit, i.e., $q \rightarrow-1$ one of them simplifies substantially, and using the fact that all the nonvanishing components of the DCF are equal, we find the same closed formula for all of them in this limit.

Let us recall the definition of one of the components of the DCF in the case of the XXZ model. Up to an overall conventional normalization factor it is given by

$$
S^{i,+-}(w, k)=\int_{-\infty}^{\infty} d t \sum_{n \in \mathbb{Z}} e^{i(w t+k n)}<0\left|\sigma^{+}(t, n) \sigma^{-}(0,0)\right| 0>_{i}
$$

here $w$ and $k$ are the energy and momentum transfer respectively, and $i$ corresponds to the boundary condition. Later we will find that the DCF is in fact independent of $i$. Using the completeness relation, the two-spinon contribution is given by

$$
\begin{aligned}
S_{2}^{i,+-}(w, k)= & \pi \sum_{n \in \mathbb{Z}} \sum_{\epsilon_{1}, \epsilon_{2}} \oint \frac{d \xi_{1}}{2 \pi i \xi_{1}} \frac{d \xi_{2}}{2 \pi i \xi_{2}} \exp \left(i n\left(k+p\left(\xi_{1}\right)+p\left(\xi_{2}\right)\right)\right) \delta\left(w-e\left(\xi_{1}\right)-e\left(\xi_{2}\right)\right) \\
& \times_{i+n}<0\left|\sigma^{+}(0,0)\right| \xi_{2}, \xi_{1}>_{\epsilon_{2}, \epsilon_{1} ; i+n} \quad i ; \epsilon_{1}, \epsilon_{2} \\
& <\xi_{1}, \xi_{2}\left|\sigma^{-}(0,0)\right| 0>_{i} .
\end{aligned}
$$

This can be re-written as

$$
\begin{aligned}
& S_{2}^{i,+-}(w, k)=\pi \sum_{\epsilon_{1}, \epsilon_{2}} \oint \frac{d \xi_{1}}{2 \pi i \xi_{1}} \frac{d \xi_{2}}{2 \pi i \xi_{2}} \sum_{n \in \mathbb{Z}} \exp \left(2 i n\left(k+p\left(\xi_{1}\right)+p\left(\xi_{2}\right)\right)\right) \delta\left(w-e\left(\xi_{1}\right)-e\left(\xi_{2}\right)\right) \\
& \times\left({ }_{i}<0\left|\sigma^{+}(0,0)\right| \xi_{2}, \xi_{1}>_{\epsilon_{2}, \epsilon_{1} ; i} \quad i ; \epsilon_{1}, \epsilon_{2}<\xi_{1}, \xi_{2}\left|\sigma^{-}(0,0)\right| 0>_{i}\right. \\
& +\exp \left(i\left(k+p\left(\xi_{1}\right)+p\left(\xi_{2}\right)\right)_{1-i}<0\left|\sigma^{+}(0,0)\right| \xi_{2}, \xi_{1}>_{\epsilon_{2}, \epsilon_{1} ; 1-i} i ; \epsilon_{1}, \epsilon_{2}<\xi_{1}, \xi_{2}\left|\sigma^{-}(0,0)\right| 0>_{i}\right) \text {. }
\end{aligned}
$$

The non-vanishing form factors have been computed in [13, and satisfy the following relations:

$$
\begin{aligned}
{ }_{i}<0\left|\sigma^{-}(0,0)\right| \xi_{2}, \xi_{1}>_{++; i} & ={ }_{1-i}<0\left|\sigma^{+}(0,0)\right| \xi_{2}, \xi_{1}>_{--; 1-i}, \\
{ }_{i}<0\left|\sigma^{-}(0,0)\right| \xi_{2}^{*}, \xi_{1}^{*}>_{++; i} & ={ }_{i}<0\left|\sigma^{+}(0,0)\right|-q \xi_{1},-q \xi_{2}>_{--; i}, \\
i_{i--}<\xi_{1}, \xi_{2}\left|\sigma^{-}(0,0)\right| 0>_{i} & ={ }_{i}<0\left|\sigma^{-}(0,0)\right|-q \xi_{1},-q \xi_{2}>_{++; i}
\end{aligned}
$$

Now the isotropic limit $q \rightarrow-1$ is performed by first making the following redefinitions:

$$
\begin{aligned}
& \xi=i e^{\frac{\epsilon \beta}{i \pi}}, \\
& q=-e^{-\epsilon}, \quad \epsilon \rightarrow 0^{+} .
\end{aligned}
$$

Here $\beta$ is the appropriate spectral parameter for the Heisenberg model. 
Then, one finds the following exact isotropic limits [13]:

$$
\begin{aligned}
& \left.\right|_{i}<0\left|\sigma^{+}(0,0)\right| \xi_{2}, \xi_{1}>\left._{--; i}\right|^{2} \frac{d \xi_{1}}{2 \pi i \xi_{1}} \frac{d \xi_{2}}{2 \pi i \xi_{2}} \rightarrow \frac{\Gamma(3 / 4)^{2}\left|A_{-}\left(\beta_{1}-\beta_{2}\right)\right|^{2}}{16 \Gamma(1 / 4)^{2}\left|A_{+}(i \pi / 2)\right|^{2}\left|A_{-}(i \pi / 2)\right|^{2} \cosh \left(\beta_{1}\right) \cosh \left(\beta_{2}\right)} d \beta_{1} d \beta_{2}, \\
& p(\xi) \rightarrow p(\beta), \quad \text { s.t. } \quad \cot (p(\beta))=\sinh (\beta), \quad-\pi \leq p(\beta) \leq 0, \\
& e(\xi) \rightarrow e(\beta)=\frac{\pi}{\cosh (\beta)}=-\pi \sin (p(\beta)),
\end{aligned}
$$

where

$$
\left|A_{ \pm}(\alpha)\right|^{2}=\exp \left(-\int_{0}^{\infty} d x \frac{\left(\cosh \left(2 x\left(1-\frac{\delta}{\pi}\right)\right) \cos \left(\frac{2 x \gamma}{\pi}\right)-1\right) \exp (\mp x)}{x \sinh (2 x) \cosh (x)}\right) .
$$

Here $\Gamma(x)$ is the usual gamma function and $\alpha=\gamma+i \delta$, with $\gamma$ and $\delta$ being real. Restricting to the first Brillouin zone, integrating the continuous and discrete delta functions, keeping track of the Jacobian factors, the energy-momentum conservation relations, and the isotropic limits of (13), we find that $S_{2}^{i,+-}(w, k)$ is independent of $i$ (which is henceforth omitted) and simplifies substantially to:

$$
S_{2}^{+-}(w, k)=\frac{\pi^{2} \Gamma(3 / 4)^{2} \Theta(2 \pi \sin (k / 2)-w) \Theta(w-\pi|\sin (k)|)}{4 \Gamma(1 / 4)^{2}\left|A_{-}(i \pi / 2)\right|^{2}\left|A_{+}(i \pi / 2)\right|^{2}} \frac{\left|A_{-}\left(\bar{\beta}_{1}-\bar{\beta}_{2}\right)\right|^{2}}{\sqrt{(2 \pi \sin (k / 2))^{2}-w^{2}}},
$$

where $\Theta$ is the Heaviside step function, and for fixed $w$ and $k, \bar{\beta}_{1}$ and $\bar{\beta}_{2}$ are the solutions to:

$$
\begin{aligned}
w & =e\left(\bar{\beta}_{1}\right)+e\left(\bar{\beta}_{2}\right), \\
k & =-p\left(\bar{\beta}_{1}\right)-p\left(\bar{\beta}_{2}\right) .
\end{aligned}
$$

Note that the pair $\left(\bar{\beta}_{1}, \bar{\beta}_{2}\right)$ is identified with the pair $\left(\bar{\beta}_{2}, \bar{\beta}_{1}\right)$.

Let us now make some comments about $S_{2}^{+-}(w, k)$ as given by (17). From the isotropy of the Heisenberg model and the inclusion of both sectors $i=0$ and $i=1$, we obtain all the non-vanishing components of the DCF from $S_{2}^{+-}(w, k)$ as:

$$
S_{2}^{x x}(w, k)=S_{2}^{y y}(w, k)=S_{2}^{z z}(w, k)=4 S_{2}^{+-}(w, k),
$$

with

$$
\sigma^{ \pm}=\frac{\sigma^{x} \pm i \sigma^{y}}{2}
$$

Furthermore, from the dispersion relations of two spinons, $w$, as a function of $k$, lies between two boundaries: the lower one is given by the famous des Cloizeaux-Pearson dispersion relation, i.e.,

$$
w_{l}=\pi|\sin (k)|,
$$


whereas the upper one is given by the dispersion relation

$$
w_{u}=2 \pi \sin (k / 2)
$$

Note that despite its square root singularity, $S_{2}^{+-}(w, k)$ actually vanishes in the vicinity of the upper boundary. Moreover, it diverges in the vicinity of the lower boundary. It would be very interesting to compare our results with presently existing approximate results, and especially the ansatz made for the two-spinon DCF in Ref. [5]. In this regard, let us mention that unlike in the latter reference, the upper cutoff at $w=w_{u}$ appears naturally in our formula. It would also be interesting to investigate the order of contribution of more than two spinons to the DCF, and in particular that of four spinons. Also of interest is to find to what extent the two-spinon DCF can satisfy the various sum rules which involve the full DCF [5]. The extension of this work to the Heisenberg model with higher spin is certainly desirable. In this case, the form factors can in principle be computed through the bosonization of the vertex operators which is now available in [14]. The full details of this paper and the relations with existing approximate results and exact results of other models will appear elsewhere. We hope that the analysis in this paper will make it convincing that infinite-dimensional symmetries, and in particular infinite-dimensional quantum groups might be of great usefulness in tackling some of the problems that have so far remained intractable and elusive through traditional methods. Finally, we hope that this work will also help improve interpretations of experimental and numerical data through the spinon picture. 


\section{Aknowlegements}

The work of A.H.B. is supported by the NSF Grant \# PHY9309888. We are grateful to Korepin and Weston for interesting discussions at early stages of this work. We are particularly thankful to Karbach and Müller for a stimulating discussion on the overall coefficient in formula (17). We highly appreciate the help from Aurag, Kidonakis, Mateev and Smith regarding numerical methods of integrations. We are also indebted to Klumper, McCoy, Perk, Sebbar, Shrock, Smirnov, and Takhtajan for illuminating discussions. 


\section{References}

[1] Th. Niemeijer, Physica 36, 377 (1967).

[2] R. J. Baxter, Exactly Solved Models in Statistical Mechanics, Academic London Press, (1982).

[3] V.E. Korepin, A.G. Izergin, and N.M. Bogoliubov, Quantum Inverse Scattering Method and Correlation Functions, Cambridge University Press, (1993).

[4] J.M.R. Roldan, B.M. McCoy and J.H.H. Perk, Physica 136A, 255 (1986).

[5] G. Müller, H. Thomas, H. Beck, and J.C. Bonner, Phys. Rev. B24, 1429 (1981).

[6] G. Müller and R. Shrock, J. Appl. Phys. 55, 1874 (1984).

[7] I. Affleck, J. Phys: Cond. Mat. 1, 3047 (1989).

[8] D.A. Tennant, R.A. Cowley, S.E. Nagler, and A. M. Tsvelik, Phys. Rev. B52, 13368 (1995).

[9] D.A. Tennant, S.E. Nagler, D.Welz, G. Shirane, and K. Yamada, Phys. Rev. B52, 13381 (1995).

[10] L.A. Takhtajan and L.D. Faddeev, Russ. Math. Surveys 34, 11 (1979).

[11] F.A. Smirnov, Form Factors in Completely Integrable Models of Quantum Field Theory, World Scientific, Singapore, (1992).

[12] O. Davies, O. Foda, M. Jimbo, T. Miwa, and A. Nakayashiki, Comm. Math. Phys. 151, 89 (1993).

[13] M. Jimbo and T. Miwa, Algebraic Analysis of Solvable Lattice Models, American Mathematical Society, (1994).

[14] A.H. Bougourzi, Journal of Physics A28, 5831 (1995). 